\title{
Effects of stimulus orientation on the identification of common polyoriented objects
}

\author{
E. C. LEEK \\ University of Wales, Bangor, Gwynedd, Wales
}

\begin{abstract}
Experimental evidence has shown that the time taken to recognize objects is often dependent on stimulus orientation in the image plane. This effect has been taken as evidence that recognition is mediated by orientation-specific stored representations of object shapes. However, the factors that determine the orientation specificity of these representations remain unclear. This issue is examined using a word-picture verification paradigm in which subjects identified line drawings of common mono- and polyoriented objects at different orientations. A detailed analysis of the results showed that, in contrast to mono-oriented objects, the recognition of polyoriented objects is not dependent on stimulus orientation. This interaction provides a further constraint on hypotheses about the factors that determine the apparent orientation specificity of stored shape representations. In particular, they support previous proposals that objects are encoded in stored representations at familiar stimulus orientations.
\end{abstract}

It is well documented that the time taken to recognize objects is often dependent on the orientation of the stimulus in the image plane-that is, a plane perpendicular to the line of sight (e.g., Arguin \& Leek, 1995; Jolicoeur, 1985; Maki, 1986; McMullen \& Farah, 1991; Murray, Jolicoeur, McMullen, \& Ingleton, 1993; Rock, 1973; Schwartz, 1981; Tarr \& Pinker, 1989). However, the theoretical implications of this effect remain the subject of considerable debate (see, e.g., Biederman \& Gerhardstein, 1993; Corballis, 1988; Leek, 1996; Tarr \& Pinker, 1990; Wagemans, Van Gool, \& Lamote, 1996).

One important issue concerns the functional origin of orientation effects in recognition tasks. For example, it could be that these effects actually result from the operation of mental processes that play no direct role in stimulus identification, such as the preparation and planning of motor actions or the calculation of stimulus orientation for the purposes of reaching and grasping even when such actions are not required by the task (see, e.g., Deutsch, Bourbon, Papanicolaou, \& Eisenberg, 1988; Georgopoulos, Lurito, Petrides, Schwartz, \& Massey, 1989). However, it is more generally assumed that they result from processes that directly support recognition. One prominent version of this account is that they reflect the use of a spatial normalization process that transforms a

This research was part of the work presented in a $\mathrm{PhD}$ thesis by E.C.L. at Johns Hopkins University, Baltimore, September 1996. Preparation of the paper was partially supported by a postdoctoral fellowship awarded by the Agence Rhone-Alpes pour les Sciences Sociales et $\mathrm{Hu}$ maines, France, held at the Université Pierre Mendes France, Grenoble. Special thanks to Brenda Rapp, Michael McCloskey, and Steven Yantis for their advice, and to James Cutting, Rebecca Lawson, Christian Marendaz, Stephen Monsell, Marie-Josèphe Tainturier, Johan Wagemans, and an anonymous reviewer for their comments on an earlier version of the manuscript. Correspondence should be addressed to E. C. Leek, Department of Psychology, University of Wales, Bangor, Gwynedd LL57 2DG, Wales (e-mail: pss034@bangor. ac.uk). perceptual representation of a stimulus to match a stored orientation-specific representation of object shape held in long-term memory (e.g., Bülthoff \& Edelman, 1992; Edelman \& Bülthoff, 1992; Jolicoeur, 1985; Rock, 1973; Tarr, 1995; Tarr \& Bülthoff, 1995; Tarr \& Pinker, 1989; Ullman, 1989). Some evidence in support of this possibility is that orientation effects seem to be influenced by stored object-specific knowledge. Thus, the time taken to recognize mono-oriented objects like cars and buildings, which are almost always seen at the same orientation in the environment, generally increases as a function of the angular distance between the orientation of the stimulus and the object's familiar upright orientation (see, e.g., Jolicoeur, 1985; McMullen \& Farah, 1991; Rock, 1973). If orientation effects arise from processes that play no role in stimulus identification, it is not clear why they should be influenced by stored knowledge about the familiar orientation of particular objects. On the other hand, several theoretical issues related to the orientation-specific representations hypothesis remain unresolved. One important question concerns the kinds of information about object orientation that such representations are likely to encode.

One proposal is that they encode shape descriptions at their familiar canonical orientation (e.g., Rock, 1973; Tarr, 1995; Tarr \& Bülthoff, 1995; Tarr \& Pinker, 1989; Ullman, 1989). Although this is consistent with the finding that mono-oriented objects are aligned to a single familiar orientation, the generality of this hypothesis is challenged by reports showing that stimulus identification is not always fastest at familiar stimulus orientations (Biederman \& Gerhardstein, 1993; Murray et al., 1993; Takano, 1989; Tarr \& Pinker, 1990; Wiser, 1981). For example, Wiser found that elongated novel shapes were recognized fastest when presented at a vertical orientation, regardless of the orientation at which they had previously been memorized. This suggests that recognition may 
sometimes be mediated by stored representations that encode shape descriptions at a canonical orientation regardless of the familiarity of that orientation (Palmer, 1989; Wiser, 1981).

This possibility raises an interesting question about how shape descriptions of common polyoriented objects are encoded in long-term memory-that is, objects like hammers, razors, and pencils that are frequently seen at several different orientations in the environment (Gibson \& Robinson, 1935; Newell \& Findlay, 1992). Most previous studies of orientation effects with common objects have been restricted to the mono-oriented object class. However, a detailed analysis of the effects of stimulus orientation on the identification of common polyoriented objects may also provide important constraints on hypotheses about the kinds of stored representations that mediate recognition. In particular, if long-term memory stores shape representations at a canonical orientation regardless of familiarity, we might expect polyoriented objects to also be aligned to a single orientation during stimulus identification. In contrast, if the orientation specificity of stored representations is determined by familiarity, we would not expect polyoriented objects to be encoded at a canonical orientation since, by hypothesis, they are likely to have several equally familiar orientations. In addition, such an analysis may provide further evidence about the functional origin of orientation effects. If the effects that have previously been observed with mono-oriented objects result solely from the operation of mental processes that play no direct role in stimulus identification, we might expect these processes to be applied to all objects regardless of their orientability. This predicts that similar orientation effects should be observed for both mono- and polyoriented objects.

These two issues were examined in this paper by comparing the effects of stimulus orientation on the recognition of mono- and polyoriented objects using a wordpicture verification paradigm. In this task, subjects judged whether written names of objects matched line drawings presented at different orientations in the image plane. This paradigm has been used in several previous recognition studies (e.g., Hamm \& McMullen, 1998; Kosslyn et al., 1994) and was chosen for two main reasons. First, in name production tasks such as picture naming, verbal responses across subjects to specific objects may vary considerably among the superordinate-, basic-, and subordinate-level names. This may be important because prior evidence suggests that orientation effects depend on the level of stimulus classification required by the task (e.g., Biederman \& Gehardstein, 1993; Hamm \& McMullen, 1998). In the word-picture verification paradigm, the level of stimulus classification required can be more carefully controlled. ${ }^{1}$ Second, in name production tasks, the selection of picture stimuli and the reliability of betweengroup comparisons are additionally constrained by lexical variables that are normally assumed to be irrelevant for object recognition (e.g., name frequency, word length, and word-initial phoneme). These variables are less important in word-picture verification tasks since task performance is not measured in terms of name production latencies.

\section{METHOD}

\section{Subjects}

Ninety-six native French-speaking subjects from the Université Pierre Mendes France, Grenoble, participated in the word-picture verification experiment for course credit. All subjects had normal or corrected-tonormal eyesight.

\section{Materials}

The stimuli were 96 black-and-white line drawings of common objects that each possessed a clearly defined principal axis of elongation. The set included 24 mono-oriented and 24 polyoriented objects selected on the basis of a prior ratings study, described below.

Although the distinction between mono- and polyoriented objects is reasonably clear on an intuitive basis, the ratings study was carried out to obtain a more objective measure of orientability. The study was carried out at Johns Hopkins University, Baltimore, using 2 subjects who were trained to record the number of different orientations at which they saw 70 familiar objects in the natural environment. The observations were recorded in a response booklet containing a picture of each stimulus depicted at eight orientations $45^{\circ}$ apart within a reference frame containing an $x$ - and a $y$-axis. The subjects were told that the $x$-axis corresponded to the direction of the gravitational vertical, and the $y$-axis to an imaginary axis perpendicular to the gravitational vertical. They were then given extensive practice recording the orientation of the principal shape axes of different objects relative to this reference frame. Data collection was carried out over a 2 -week period. The subjects were told that whenever they saw one of the test objects in the natural environment they should record the approximate orientation of its principal shape axis relative to the closest of the eight orientations depicted in the response booklet. ${ }^{2}$

At the end of the 2-week period, the number of times each object had been seen by either subject at one of the orientations was calculated. Twenty-four objects that had been seen at only one orientation were selected for use as mono-oriented stimuli in the word-picture verification experiment. The 24 objects that had been seen at the highest number of different orientations were chosen as polyoriented stimuli. The mean number of different orientations at which the polyoriented objects had been seen was $6.75 / 8(S D=1.33)$. A list of the orientation ratings for each stimulus is shown in Appendix A.

For the word-picture verification experiment, a line drawing of each stimulus was digitized using a scanner and recopied in a $12 \times 12 \mathrm{~cm}$ frame at the following eight orientations in the image plane: $0^{\circ}, 45^{\circ}$, $90^{\circ}, 135^{\circ}, 180^{\circ}, 225^{\circ}, 270^{\circ}$, and $315^{\circ}$. The $0^{\circ}$ orientation of the monooriented objects always corresponded to their normal upright orientation with respect to the gravitational vertical. Thus, this orientation was vertical for some objects (e.g., skyscraper, lamp) and horizontal for others (car, motorcycle). The $0^{\circ}$ orientation of the polyoriented objects was always such that the principal axis of elongation was parallel to the vertical axis of the monitor.

\section{Apparatus}

The experiment was run on a Macintosh IIci computer, using Psychlab Version 0.91 software (Gum, 1995).

\section{Design}

There were 14 practice trials followed by 96 experimental trials. On each trial the written basic-level French name of an object (e.g., arbretree, voiture-car) was presented briefly on the screen and then replaced by a picture. There were two types of trials. On yes response trials, the name was appropriate for the picture that followed it. On no response trials, the name denoted another object type that was visually, and where possible, semantically similar to the picture (e.g., banjo $\rightarrow$ guitar $r$ ). This 
was done to ensure that the subjects could not make accurate responses solely by considering either the global shape outlines of the objects or specific individual shape features. The mono- and polyoriented objects chosen from the orientability ratings study were shown only in the yes response trials. The stimuli for the no response trials were randomly selected pictures of elongated common objects. In total, there were 48 yes ( 24 mono-oriented and 24 polyoriented) and 48 no response trials. Since orientation effects in recognition tasks are known to attenuate with practice (see, e.g., Jolicoeur, 1985; Murray et al., 1993), the subjects saw each stimulus only once. In total, each object was seen at each stimulus orientation by 12 different subjects. The trials were randomized prior to the beginning of the experiment.

\section{Procedure}

The subjects were seated approximately $36 \mathrm{~cm}$ from the monitor. A chinrest was used to prevent inclinations of the head. Each trial began with a prompt that remained in the center of the screen until the subject pressed the space bar. After a blank interval of $500 \mathrm{msec}$, the written name of an object was displayed for $750 \mathrm{msec}$ in the same location as the prompt followed by another blank interval of $250 \mathrm{msec}$. After this, a line drawing of an object was presented in the center of the screen at one of eight orientations in the image plane. The line drawing remained on the screen until the subject made a response. The task was to respond "yes" if the written name was appropriate for the picture and "no" if it was not. There was a response deadline of $2,500 \mathrm{msec}$. If the subjects did not respond before the deadline, they heard an error tone. Responses were made by pressing one of two keys on a standard keyboard labeled "oui" (yes) and "non" (no). The yes responses were always made with the dominant hand. The experiment lasted about $20 \mathrm{~min}$.

\section{RESULTS AND DISCUSSION}

\section{Analysis of Reaction Times}

Preliminary analyses were performed by subjects and items on the mean median reaction times (RTs) for correct responses in the yes trials only. Figure 1 shows the mean median RTs across stimulus orientations for the mono- and polyoriented objects.

A two-way analysis of variance (ANOVA) by subjects with stimulus orientation and object type (mono- vs. polyoriented) as factors showed a significant main effect of stimulus orientation $[F(7,665)=3.39, p<.001]$ and object type $[F(1,95)=70.46, p<.001]$, as well as a significant interaction $[F(7,665)=2.39, p<.01]$. An analysis of simple effects showed that there was a significant effect of stimulus orientation for the mono-oriented objects $[F(7,665)=4.33, p<.001]$, but not the polyoriented objects $[F(7,665)=1.5]$. A two-way ANOVA by items showed a significant main effect of stimulus orientation $[F(7,161)=2.70, p<.01]$ and a significant interaction $[F(7,161)=3.75, p<.001]$, but the main effect of object type just failed to reach significance $[F(1,23)=3.45, p<$ .07]. An analysis of simple effects confirmed that there was a significant effect of stimulus orientation for the mono-oriented objects $[F(7,161)=6.59, p<.001]$, but not for the polyoriented objects $[F(7,161)=0.67]$.

Additional analyses using the $t$ test for related groups showed that there were no significant differences between the RTs for mono-oriented objects presented at their upright canonical orientation and the RTs for the polyoriented objects presented at any of the eight stimulus orientations. This suggests that the absence of an orientation effect for the polyoriented objects cannot be solely accounted for in terms of a ceiling or floor effect.

An analysis was also performed to examine whether the pattern of RTs for the mono-oriented objects increased as a function of the angular distance of the stimuli from their familiar canonical orientation, as shown in several previous reports (e.g., Jolicoeur, 1985). In order to examine this possibility, the median RTs at each stimulus orientation, excluding $180^{\circ}$, were regressed against their angular distance from the $0^{\circ}$ orientation. ${ }^{3}$ The results showed that within this range of orientations, distance from the canonical orientation accounted for a significant amount of variance in the RTs for the mono-oriented objects

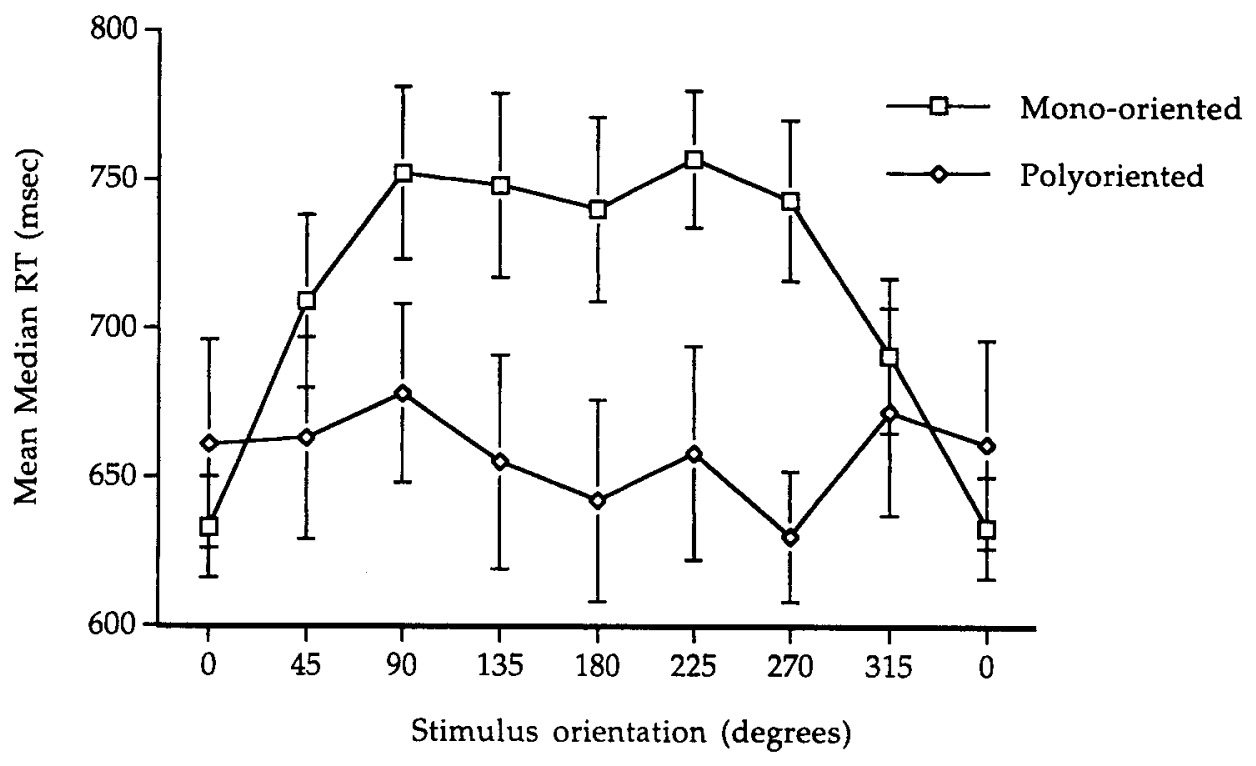

Figure 1. Mean median reaction times (RTs) for the mono- and polyoriented objects as a function of stimulus orientation. Bars indicate standard error. RTs at the $0^{\circ}$ orientation are repeated for ease of comparison. 
$\left[r^{2}=.84 ; F(1,6)=27.16, p<.003\right]$, but not the polyoriented objects $\left[r^{2}=.06 ; F(1,6)=0.31\right]$. Although this analysis is relatively weak, since it is based on RTs across only seven orientations, it does distinguish between the different patterns observed for the mono- and polyoriented objects.

\section{Analysis of Error Rates}

An analysis of error rates showed that the subjects made $394 / 4,604(8.55 \%)$ errors on no response trials and 191/ $4,604(4.14 \%)$ errors on yes response trials. A Wilcoxon test showed that this difference was significant $\left[Z_{\text {corr }}=\right.$ $-6.51, p<.001]$. For the yes responses, the subjects made $73 / 2,302(3.17 \%)$ errors on mono-oriented object trials and $118 / 2,302(5.12 \%)$ errors on polyoriented object trials, although this difference was not significant. The percentage error rates in the yes response trials as a function of stimulus orientation for both object types is shown in Figure 2.

A two-way ANOVA on error rates for the yes response trials with stimulus orientation and object type as factors showed that there were no main effects of either factor and no significant interaction. This suggests that the pattern of RTs for the mono- and polyoriented objects cannot be solely accounted for in terms of a speed -accuracy tradeoff.

\section{Additional Planned Analyses of Polyoriented Object RTs}

The results presented so far are consistent with previous reports that the time taken to identify mono-oriented objects is dependent on the angular distance of the stimulus from the canonical orientation. In contrast, the time taken to identify polyoriented objects does not appear to be dependent on stimulus orientation. However, this finding shows only that polyoriented objects are not aligned to a single arbitrarily determined image plane orientation at which the principal axis of elongation is parallel with the visual vertical. The preliminary analyses do not address the possibility that polyoriented objects are aligned to some other single orientation.

One possibility is that these objects are aligned to a single orientation that is consistent across subjects but different across objects. If this were the case, we would expect the pattern of RTs for individual polyoriented objects to show an alignment of the stimulus to a single orientation-as observed for the mono-oriented objects. Since the canonical orientation may vary across objects, these alignment effects may have been obscured in the preliminary analyses by averaging RTs across objects. This possibility was examined by considering the pattern of RTs for the polyoriented objects on an item-by-item basis. For each object, the stimulus orientation with the fastest RT was assumed to be the canonical orientation. This orientation was recoded as the $0^{\circ}$ orientation and adjacent cells were recoded as a function of their angular distance from the canonical orientation. For example, if the fastest RT for the pencil was at $90^{\circ}$, this orientation would be recoded as the $0^{\circ}$ orientation and the RT at $135^{\circ}$ recoded as the $45^{\circ}$ orientation, and so on. Once the data for each object had been recoded in this way, the pattern of mean median RTs across stimulus orientations was recalculated. A one-way ANOVA on the realigned data showed that there was a significant effect of stimulus orientation for the polyoriented objects $[F(7,322)=10.4$. $p<.001]$. However, this effect is not surprising given that the $0^{\circ}$ orientation now contained only the fastest RTs and had an artificially low mean. The crucial question is

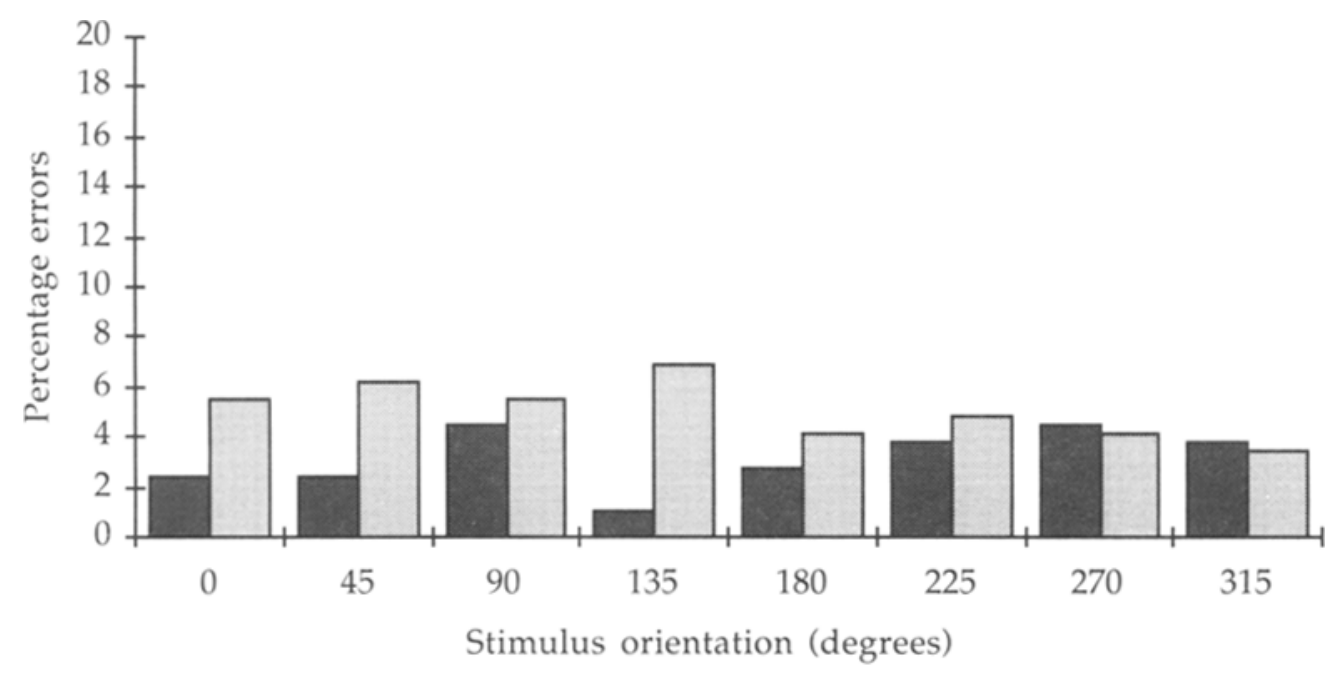

- Mono-oriented a Polyoriented 
whether the pattern of RTs across stimulus orientations increases as a function of the angular distance between the orientation of the stimulus and the putative canonical orientation, as was previously observed for the monooriented objects. A simple regression analysis showed that this was not the case: The angular distance of the stimulus from the $0^{\circ}$ orientation was not a significant predictor of RTs $\left[r^{2}=.28, F(1,6)=1.95, p<.22\right]$.

For comparison, the same recoding procedure was applied to the RT data for the mono-oriented objects. A oneway ANOVA on the recoded data showed that there was a significant effect of stimulus orientation $[F(7,322)=$ $13.6, p<.001]$. A simple regression analysis also confirmed that the angular distance of the stimulus from the canonical orientation remained a significant predictor of RTs $\left[r^{2}=.80 ; F(1,6)=24.57, p<.01\right]$. Thus, the results of these analyses are inconsistent with the possibility that polyoriented objects are aligned to a single orientation that is the same across subjects but different across objects.

Another possibility that was not examined in the preliminary analyses is that polyoriented objects might be aligned to a single orientation that varies across both subjects and objects (Cutzu \& Edelman, 1994). One way to examine this possibility would be to consider the patterns of RTs across stimulus orientations for each subject and item separately. However, since each subject saw each item only once, this analysis was not possible. Instead, an alternative analysis was performed on the basis of the following prediction: If the canonical orientation of polyoriented objects varies both across subjects and objects, we would expect the variance in RTs for each object at each stimulus orientation (i.e., within each cell) to be larger for the polyoriented objects than for the monooriented objects. This is because for any given stimulus orientation, the angular distance to the canonical orientation should vary across subjects for the polyoriented objects but be the same across subjects for the monooriented objects. ${ }^{4}$ This prediction was tested by calculating the standard deviation in RTs at each stimulus orientation for each item separately-that is, in all 384 cells ( 48 objects $\times 8$ orientations). The mean standard deviation per cell for the mono-oriented objects was $292 \mathrm{msec}$ $(S D=141 \mathrm{msec})$ and for the polyoriented objects it was $281 \mathrm{msec}(S D=167 \mathrm{msec})$. A $t$ test for related groups showed that this difference was not significant $[t(1,191)=$ .69]. The results of this analysis are inconsistent with the possibility that polyoriented objects are aligned to a single orientation that varies across both subjects and objects.

\section{Further Issues}

It has been assumed that the crucial distinction between the two object types contrasted in this experiment is orientability - that is, the number of different orientations at which the objects are normally seen in the environment. However, one might argue that the mono- and polyoriented stimuli also differ systematically in other respects. First, the mono-oriented objects generally tend to be physically larger than the polyoriented objects. Previous studies on mental imagery suggest that different stimulus properties may influence mental rotation performance (e.g., Kosslyn, 1973; Kosslyn \& Alper, 1977). Thus, one might argue that the observed patterns of RTs actually reflect an interaction of object size and rotation rate. Second, the line drawings of the mono- and polyoriented stimuli might also differ systematically in terms of their compactness - that is, the ratio of contour perimeter and area. On some hypotheses, this factor has been assumed to influence recognition performance (e.g., Zusne, 1970). For example, it may be that pictorial compactness affects how quickly the orientation of the principal shape axis of the stimulus could be computed. Thus, the observed interaction in RTs may actually depend on pictorial compactness rather than orientability. The following post hoc analyses were carried out to address these possibilities. ${ }^{5}$

\section{Effects of Physical Size}

The effect of physical size was examined in the following manner: First, 10 additional subjects were presented with a randomized list containing the names of the 24 mono- and 24 polyoriented objects used in the wordpicture verification experiment. They were asked to rate the relative volumetric size of each object using a scale in which the largest object (skyscraper) was preassigned a value of 10,000 , and the smallest object (screw) a value of 1. A physical size index was calculated by taking the mean ratings across subjects for each object. Second, an RT index for each stimulus was calculated using the data from the word-picture verification experiment. This was done by subtracting the median RTs for each item at the $0^{\circ}$ orientation from the average RT across stimulus orientations between $45^{\circ}$ and $315^{\circ}$. The physical size and RT indices for each stimulus are shown in Appendix A.

The effect of physical size was examined using a multiple regression analysis on data from the 23 mono- and 23 polyoriented objects for which physical size ratings were obtained. In the analysis, two predictor variablesthe physical size index for each object and a dummy code for orientability - were regressed against the RT index. The results showed that the two predictor variables accounted for a significant amount of variance in the RT index $\left[r_{\text {multiple }}^{2}=.261 ; F(2,43)=7.62, p<.001\right]$. Analyses using partial correlation showed that the amount of variance accounted for solely by orientability with physical size partialed out was significant $\left[\mathrm{Corr}_{\text {partial }}=-.48\right.$; $t(43)=-3.62, p<.001]$, but physical size alone was not a significant factor $\left[\operatorname{Corr}_{\text {partial }}=-.06, t(43)=-.40\right]$.

\section{Effects of Pictorial Compactness}

The effect of pictorial compactness was examined by first calculating the compactness value for each mono- and polyoriented stimulus using the formula compactness = $\left(\mathrm{P}^{2} / \mathrm{A}\right)$, where $\mathrm{P}$ is the length of the perimeter contour of the image and $A$ is the area (Zusne, 1970). The compact- 
ness value for each object together with dummy codes for orientability were then regressed against the RT index. As previously, the results showed that the two predictor variables accounted for a significant amount of variance in the RT index $\left[r^{2}\right.$ multiple $=.30 ; F(2,45)=9.64, p<$ $.001]$. Partial correlations showed that the amount of variance accounted for solely by orientability with compactness partialed out was significant $\left[\right.$ Corr $_{\text {partial }}=-.49$; $t(45)=-3.78, p<.001]$, but compactness alone was not a significant factor $\left[\operatorname{Corr}_{\text {partial }}=-.15 ; t(43)=-.30\right]$.

These post hoc analyses show that differences in the pictorial compactness and physical size between the monoand polyoriented objects do not contribute to the interaction of RTs across stimulus orientations observed in the word-picture verification experiment.

\section{GENERAL DISCUSSION}

These results, consistent with several previous reports, show that the time taken to identify line drawings of common mono-oriented objects increases as a function of the angular distance between the orientation of the stimulus and its familiar canonical orientation. In contrast, the time taken to identify common polyoriented objects seems to be independent of their image plane orientation. Further analyses support the view that this interaction derives from differences in the orientability of mono- and polyoriented objects rather than other stimulus properties that might be expected to influence the time taken to identify misoriented objects such as pictorial compactness or physical size

In the first place, it is necessary to consider one potential challenge to the importance of these results on the grounds that the word-picture verification task used in this experiment has little relevance for hypotheses about the mental processes and shape representations that mediate everyday object recognition. In response to this challenge, it is important to note that a similar orientation effect was observed for the mono-oriented objects in this experiment, as has previously been observed in several other studies using, arguably, more direct recognition tasks such as picture naming (e.g., Jolicoeur, 1985). The similarity of these effects from different experimental paradigms provides some motivation for assuming that the word-picture verification task taps functional processes that are ordinarily recruited during object recognition. Consequently, it is suggested that these results provide new evidence both about the functional origin of orientation effects and about the kinds of stored representations that mediate object recognition.

First, the results are inconsistent with the hypothesis that orientation effects in recognition tasks result from mental processes that are applied to all objects but that play no direct role in stimulus identification. If this were the case, we would expect polyoriented objects to show similar orientation effects to mono-oriented objects.

Second, the results are inconsistent with the possibility that recognition is mediated by stored representations that encode shape descriptions at a canonical orientation regardless of its familiarity. If this were so, we would expect polyoriented objects to be aligned to a single orientation during stimulus identification. However, the results showed that this was not the case. ${ }^{6}$

How. then, can these results be accounted for? One recent proposal that can account for these results is the "multiple-views" hypothesis (see, e.g., Tarr, 1995; Tarr \& Bülthoff, 1995; Tarr \& Pinker, 1989). On this hypothesis, it is assumed that long-term memory encodes orientationspecific shape descriptions. The orientation specificity of the representations is determined by the orientation at which objects are encountered-. that is, by familiarity. Since mono-oriented objects are, by hypothesis, usually seen only at a single orientation, they are likely to be encoded in long-term memory at their familiar canonical orientation. However, polyoriented objects, which are often seen at several orientations, may be encoded in multiple orientation-specific representations at different familiar orientations. On some versions of this hypothesis, the recogni- tion of misoriented objects is assumed to involve a continuous spatial transformation or alignment of the stimulus to match the orientation of the corresponding stored representation (see, e.g., Tarr, 1995; Tarr \& Pinker, 1989; Ullman, 1989). Thus, the time taken to identify a misoriented mono-oriented object may be assumed to increase as a function of the angular distance between the orientation of the stimulus and its canonical orientation. In contrast, assuming that there is a sufficiently large number of different orientation-specific representations, the time taken to identify polyoriented objects may be relatively constant because the angular distance to the nearest stored representation is likely to be roughly equal regardless of stimulus orientation.

Another possibility that is consistent with the multiple-views hypothesis is that polyoriented objects are stored at only two or three different orientations. Unfortunately, the present experiment does not allow us to examine this possibility since only a relatively small number of different stimulus orientations were probed. One might also speculate about the kinds of stored representations that encode natural kinds versus artifacts. Most of the objects used in this experiment were artifacts, but certain natural kinds of objects, like cats, squirrels, and birds, are normally assumed to have a canonical orientation, although, arguably, they are not strongly mono-oriented (e.g., Palmer, Rosch, \& Chase, 1981; Rosch, 1978). ${ }^{7}$ Thus, although these results support the view that familiarity is an important determinant of the canonical image plane orientation of mono-oriented objects, this does not preclude the possibility that other factors also play a role (see, e.g., Cutzu \& Edelman, 1994; Palmer et al., 1981).

Finally, some alternative accounts of the interaction between monoand polyoriented objects must also be considered. One possibility is that polyoriented objects are encoded in orientation-invariant representations such as the object-centered structural descriptions proposed by Marr and Nishihara (1978). Another possibility is that the recognition of polyoriented objects is mediated by some kind of orientation-invariant feature identification process (see, e.g., Corballis, 1988; Humphreys \& Riddoch, 1984; Jolicoeur, 1985, 1990; Murray et al., 1993; Sutherland, 1968; Takano, 1989; Tarr, 1995; Ullman, 1989). However, although some evidence suggests that orientation-invariant recognition for image plane rotations may be achieved under certain conditions (Murray et al., 1993; Takano, 1989; Tarr \& Pinker, 1990), it is not obvious why only polyoriented objects could be encoded in object-centered representations or identified using an orientation-invariant feature-matching process. First, in terms of the hypothesis proposed by Marr and Nishihara, the polyoriented objects used in this experiment each possessed a clearly defined principal axis of elongation that should be sufficient for the computation of an axis-based, object-centered representation. Second, given the rich feature structure of most common mono- and polyoriented objects, it is not obvious why a feature-based orientationinvariant process could be applied to only one object class. Thus, as presently articulated, these alternative hypotheses do not seem to provide an entirely satisfactory account of the interaction between monoand polyoriented objects observed in this experiment.

In summary, the results reported in this paper show that, unlike common mono-oriented objects, polyoriented objects are not aligned to a single orientation during recognition. This finding provides some new evidence about the functional origin of orientation effects in recognition tasks and about the kinds of stored orientation-specific representations that mediate recognition. It is argued that these results are consistent with previous hypotheses that the recognition of polyoriented objects is mediated by multiple orientation-specific stored representations that encode information about the familiar orientations at which objects have previously been encountered.

\section{REFERENCES}

Arguin, M., \& Leek, E. C. (1995). Mental rotation for vistal object recognition: Identical rales in priming and picture naming. Poster presented at the annual meeting of the Association for Research in Vision and Ophthalmology, Fort Lauderdale, FL.

Biederman, I., \& Gerhardstein, P. C. (1993). Recognizing depthrotated objects: Evidence and conditions for three-dimensional view- 
point invariance. Journal of Experimental Psychology: Human Perception \& Performance, 19, 1162-1182.

Bülthoff, H. H., \& Edelman, S. (1992). Psychophysical support for a $2 D$ view interpolation theory of object recognition. Proceedings of the National Academy of Sciences, 89, 60-64.

Corballis, M. C. (1988). Recognition of disoriented shapes. Psychological Review, 95, 115-123.

Cutzu, F., \& Edelman, S. (1994). Canonical views in object representation and recognition. Vision Research, 34, 22, 3037-3056.

Deutsch, G., Bourbon, W. T., Papanicolaou, C., \& Eisenberg, H. M. (1988). Visuospatial experiments compared via activation of regional cerebral blood flow. Neuropsychologia, 26, 445-452.

Edelman, S., \& BülthofF, H. H. (1992). Orientation-dependence in the recognition of familiar and novel views of 3D objects. Vision Research, 32, 2385-2400.

Georgopoulos, A. P., Lurito, J. T., Petrides, M., Schwartz, A. B. \& MASSEY, J. T. $(1989)$. Mental rotation of the neuronal population vector. Science, 243, 234-236.

GibSON, J. J., \& RoBINSON, D. (1935). Orientation in visual perception: The recognition of familiar plane forms in differing orientations. Psychological Monographs, 46, 39-47.

GuM, T. (1995). Psychlab v1.0: Macintosh applications program [Software program]. Montreal: Psychlab.

Hamm, J. P., \& McMullen, P. A. (1998). Effects of orientation on the identification of rotated objects depend on the level of identity. Journal of Experimental Psychology: Human Perception \& Performance, 24, 413-426.

HumPHREYs, G. W., \& RidDOCH, M. J. (1984). Routes to object constancy: Implications from neurological impairments of object constancy. Quarterly Journal of Experimental Psychology, 36A, 385-415.

JOLICOEUR, P. (1985). The time to name disoriented natural objects. Memory \& Cognition, 13, 289-303.

Jolicoeur, P. (1990). On the role of mental rotation and feature extraction in the identification of disoriented objects: A dual systems theory. Mind \& Language, 5, 387-410.

KossLYN, S. M. (1973). Scanning visual images: Some structural implications. Perception \& Psychophysics, 14, 90-94.

KosSLYN, S. M., \& ALPER, S. N. (1977). On the pictorial properties of visual images: Effects of image size on memory for words. Canadian Journal of Psychology, 31, 32-40.

Kosslyn, S. M., Alpert, N. M., Thompson, W. L., Chabris, C. F., RAUCH, S. L., \& ANDERSON, A. K. (1994). Identifying objects seen from different viewpoints. A PET investigation. Brain, 117, 1055-1071.

LEEK, E. C. (1996). The representation of object orientation. Unpublished doctoral dissertation, Johns Hopkins University, Baltimore.

MAKI, R. H. (1986). Naming and locating the tops of rotated pictures, Canadian Journal of Psychology, 40, 368-387.

MARR, D., \& NishihaRA, H. K. (1978). Representation and recognition of the spatial organisation of three-dimensional shapes. Proceedings of the Royal Society of London: Series B, 207, 187-217.

MCMullen, P. A., \& FARAh, M. J. (1991). Viewer-centered and objectcentered representations in the recognition of naturalistic line drawings. Psychological Science, 2, 275-277.

Murray, J. E., Jolicoeur, P., McMullen, P. A., \& Ingleton, M. (1993). Orientation-invariant transfer of training in the identification of rotated natural objects. Memory \& Cognition, 21, 604-610.

NeWELL, F, \& FindLAY, J. M. (1992). Viewpoint invariance in object recognition. Irish Journal of Psychology, 13, 494-507.

PaLMer, S. E. (1989). Reference frames in the perception of shape and orientation. In B. E. Shepp \& S. Ballesteros (Eds.), Object perception: Structure and process (pp. 121-163). Hillsdale, NJ: Erlbaum.

Palmer, S. E., Rosch, E., \& Chase, P. (1981). Canonical perspective and the perception of objects. In J. Long \& A. Baddeley (Eds.), Attention and performance $I X$ (pp. 135-151). Hillsdale, NJ: Erlbaum.

Rock. I. (1973). Orientation and form. New York: Academic Press

Rosch, E. (1978). Principals of categorization. In E. Rosch \& B. B. Lloyd (Eds.), Cognition and categorization (pp. 27-48). Hillsdale, NJ: Eribaum.

SCHWARTZ, S. P. (1981). The perception of disoriented complex objects.
In Proceedings of the Third Annual Conference of the Cognitive Science Society (pp. 181-183).

Sutheriand, N. S. (1968). Outlines of a theory of visual pattern recognition in animals and man. Proceedings of the Royal Society of London: Series B, 171, 297-317.

TAKANO, Y. (1989). Perception of rotated forms: A theory of information types. Cognitive Psychology, 21, 1-59.

TARR, M. J. (1995). Rotating objects to recognize them: A case study on the role of viewpoint dependency in the recognition of three-dimensional objects. Psychonomic Bulletin \& Review, 2, 55-82.

TARR, M. J., \& BÜLTHOFF, H. H. (1995). Is human object recognition better described by geon-structural-descriptions or by multiple-views? Journal of Experimental Psychology: Human Perception \& Performance, 21, 1494-1505.

TARR, M. J., \& PINKER, S. (1989). Mental rotation and orientation dependence in shape processing. Cognitive Psychology, 21, 233-282.

TARR, M. J., \& PINKER, S. (1990). When does human object recognition use a viewer-centered reference frame? Psychological Science, 1, 253-256.

UlLMAN, S. (1989). Aligning pictorial descriptions: An approach to object recognition. Cognition, 32, 193-254.

Wagemans, J., VAN Gool, L., \& Lamote, C. (1996). The visual system's measurement of invariants need not itself be invariant. Psychological Science, 7, 232-236.

WISER, M. (1981). The role of intrinsic axes in shape recognition. In Proceedings of the Third Annual Conference of the Cognitive Science Society (pp. 184-186).

ZUSNE, L. (1970). Visual perception of form. New York: Academic Press.

\section{NOTES}

1. Of course, it is not possible to ensure that each subject accesses the same kinds of information during task performance. However, the word-picture verification paradigm does allow us to at least control the level of shape classification necessary for accurate performance (e.g., superordinate, basic, or subordinate)

2. It should be stressed that the purpose of these ratings was merely to provide a very approximate measure of stimulus orientability as a basis for selecting stimuli for use in the word-picture verification experiment. They were not intended to provide a definitive set of orientability ratings.

3. RTs for stimuli presented at $180^{\circ}$ were excluded from this analysis since previous reports have shown that they generally decrease at this orientation (i.e., when mono-oriented objects are presented upside down). A similar pattern also seems to have been present in this experiment (Figure 1). The reason why there is an M-shaped rather than an inverted U-shaped RT function remains unclear (see Biederman \& Gerhardstein, 1993; Jolicoeur, 1985; Tarr \& Pinker, 1989).

4. Another possibility would be that larger variance in the RTs for the polyoriented objects could reflect greater heterogeneity among these items in terms of others factors, such as object familiarity or visual complexity. However, since the variance was not larger for the polyoriented objects, these factors were not taken into account in the present analysis.

5. The author thanks James Cutting for raising these possibilities and suggesting the analyses.

6 . It is also interesting to note that the observation of a strong orientation effect for the mono-oriented objects in this experiment seems to be inconsistent with the finding recently reported by Hamm and McMullen (1998) that such effects appear only when the task requires subordinate-level classification (see also Biederman \& Gehardstein, 1993). In the present experiment, word-picture pairings were at a basic level. This suggests that basic-level classification, at least under certain conditions, is sufficient for producing orientation effects. However, the exact reason for these different results is not clear.

7. For example, it would be interesting to exploit image plane orientation effects as a means of examining the kinds of stored representations that mediate the recognition of these types of natural objectsthat is, whether, like artifactual mono-oriented objects, natural objects are also aligned to a single image plane orientation during recognition. 
APPENDIX A

A List of the Mono- and Polyoriented Objects Used in the Yes Response Trials Together With Their Reaction Times (RTs) and Compactness and Physical Size Values

\begin{tabular}{|c|c|c|c|}
\hline \multirow[b]{2}{*}{ Stimulus } & \multicolumn{3}{|c|}{ Index } \\
\hline & RT & Compactness & Physical Size \\
\hline \multicolumn{4}{|c|}{ Mono-oriented } \\
\hline 1 chair & 60.5 & 44.7 & 20 \\
\hline 2 lamp & 38.0 & 31.6 & 15 \\
\hline 3 computer & 331.0 & 14.4 & 41 \\
\hline 4 truck & 117.5 & 22.9 & 300 \\
\hline 5 television & 63.5 & 18.7 & 42 \\
\hline 6 motorcycle & 66.0 & 25.8 & 50 \\
\hline 7 stool & -36.5 & 49.1 & 50 \\
\hline 8 tree & 123.0 & 14.9 & 140 \\
\hline 9 car & 164.5 & 18.9 & 200 \\
\hline 10 table & 88.0 & 104.5 & 50 \\
\hline 11 fridge & 218.5 & 18.0 & 70 \\
\hline 12 bench & 184.0 & 13.0 & 50 \\
\hline 13 sailboat & 89.0 & 14.6 & 500 \\
\hline 14 washing machine & 2.5 & 16.5 & 50 \\
\hline 15 desk & 41.5 & 20.8 & 50 \\
\hline 16 wardrobe & 48.0 & 16.4 & 70 \\
\hline 17 dresser & 140.5 & 19.1 & 50 \\
\hline 18 clock & 72.0 & 29.1 & 65 \\
\hline 19 crane & 43.5 & 172.6 & 500 \\
\hline 20 church & 127.5 & 17.5 & 800 \\
\hline 21 bed & 123.5 & 24.1 & 80 \\
\hline 22 house & -50.0 & 16.2 & 500 \\
\hline 23 skyscraper & 247.5 & 30.1 & 10,000 \\
\hline 24 signpost & 98.5 & 98.6 & 43 \\
\hline Mean & 100.0 & 35.55 & $162.4^{*}$ \\
\hline \multicolumn{4}{|c|}{ Polyoriented } \\
\hline 25 pencil & 43.0 & 76.6 & 5 \\
\hline 26 lighter & 81.5 & 19.0 & 7 \\
\hline 27 razor & -173.5 & 77.3 & 5 \\
\hline 28 clothespeg & 37.5 & 71.4 & 5 \\
\hline 29 scissors & -73.0 & 146.1 & 8 \\
\hline 30 banana & -102.5 & 15.9 & 12 \\
\hline 31 tennis & 141.5 & 31.7 & 25 \\
\hline 32 comb & -77.0 & 194.6 & 8 \\
\hline 33 carrot & -11.5 & 62.5 & 9 \\
\hline 34 pen & -92.0 & 25.3 & 5 \\
\hline 35 match & 4.0 & 73.5 & 2 \\
\hline 36 nail & 16.0 & 51.9 & 2 \\
\hline 37 hammer & 143.0 & 48.9 & 15 \\
\hline 38 knife & -25.5 & 53.0 & 8 \\
\hline 39 axe & 204.0 & 47.9 & 20 \\
\hline 40 toothbrush & -80.5 & 56.5 & 8 \\
\hline $41 \mathrm{key}$ & 68.5 & 33.5 & 5 \\
\hline 42 screwdriver & -66.0 & 82.8 & 9 \\
\hline 43 pear & -127.5 & 20.3 & 10 \\
\hline 44 flashlight & 33.5 & 39.7 & 9 \\
\hline 45 lipstick & -33.5 & 24.6 & 4 \\
\hline 46 paintbrush & -70.0 & 68.3 & 9 \\
\hline 47 cigarette & -30.5 & 60.4 & 4 \\
\hline 48 screw & -278.5 & 26.3 & 1 \\
\hline Mean & -19.5 & 58.7 & $8.4^{*}$ \\
\hline
\end{tabular}

*The mean physical size excludes the preassigned values of 10,000 and 1 for skyscraper and screw, respectively. 
APPENDIX B

A List of Stimuli Used in the No Response Trials

\begin{tabular}{|c|c|}
\hline Written Name & Picture Stimulus \\
\hline 1 bagpipe & accordion \\
\hline 2 lizard & alligator \\
\hline 3 harpoon & anchor \\
\hline 4 onion & apple \\
\hline 5 leek & asparagus \\
\hline 6 wolf & bear \\
\hline 7 thermos & bottle \\
\hline 8 dragonfly & butterfly \\
\hline 9 giraffe & camel \\
\hline 10 dagger & candlestick \\
\hline 11 flute & wineglass \\
\hline 12 squirrel & cat \\
\hline 13 cucumber & celery \\
\hline 14 pheasant & chicken \\
\hline $15 \mathrm{clog}$ & shoe \\
\hline 16 bison & cow \\
\hline 17 hamster & mouse \\
\hline 18 horse & deer \\
\hline 19 pigeon & duck \\
\hline 20 mitten & glove \\
\hline 21 flamingo & swan \\
\hline 22 banjo & guitar \\
\hline 23 bonnet & hat \\
\hline 24 stocking & boot \\
\hline 25 cigar & pipe \\
\hline 26 hair-dryer & iron \\
\hline 27 egg-cup & vase \\
\hline 28 deckchair & ironing-board \\
\hline 29 fairy-light & lightbulb \\
\hline 30 tricycle & bicycle \\
\hline 31 hydroplane & helicopter \\
\hline 32 radish & mushroom \\
\hline 33 hairclip & padlock \\
\hline 34 briefcase & suitcase \\
\hline 35 bucket & watering-can \\
\hline 36 telescope & binoculars \\
\hline 37 mop & broom \\
\hline 38 metal-file & wrench \\
\hline 39 rifle & pistol \\
\hline 40 glider & airplane \\
\hline 41 hairbrush & brush \\
\hline 42 glass & cup \\
\hline 43 avocado & pepper \\
\hline 44 coconut & pineapple \\
\hline 45 coffee-pot & pitcher \\
\hline 46 tweezers & pliers \\
\hline 47 chainsaw & saw \\
\hline 48 frying-pan & saucepan \\
\hline
\end{tabular}

(Manuscript received June 2, 1997;

revision accepted for publication January 30, 1998.) 